\title{
THE DISCRETE NONLINEAR SCHRÖDINGER EQUATION - 20 YEARS ON
}

\author{
J. CHRIS EILBECK \\ Department of Mathematics, \\ Heriot-Watt University, \\ Edinburgh EH14 4AS, UK \\ E-mail: J.C.Eilbeck@hw.ac.uk \\ MAGNUS JOHANSSON \\ Dept of Physics and Measurement Techn. \\ Linköping University, \\ S-58183 Linköping, Sweden \\ E-mail:majoh@ifm.liu.se
}

We review work on the Discrete Nonlinear Schrödinger (DNLS) equation over the last two decades.

\section{Introduction}

The Discrete Nonlinear Schrödinger (DNLS) equation describes a particularly simple model for a lattice of coupled anharmonic oscillators. In one spatial dimension, the equation in its simplest form is

$$
i \frac{d A_{j}}{d t}+\gamma\left|A_{j}\right|^{2} A_{j}+\varepsilon\left(A_{j+1}+A_{j-1}\right)=0,
$$

where $i=\sqrt{-1}$, the index $j$ ranges over the $1 \mathrm{D}$ lattice. The lattice may be infinite $(j=0, \pm 1, \pm 2, \ldots)$ or finite $(j=1,2, \ldots, f)$. In the latter case one usually assumes periodic boundary conditions, $A_{j+f}=A_{j}$. The quantity $A_{j}=A_{j}(t)$ is the complex mode amplitude of the oscillator at site $j$, and $\gamma$ is a anharmonic parameter.

The connection with the continuous Nonlinear Schrödinger (NLS) equation

$$
i A_{t}+\gamma|A|^{2} A+A_{x x}=0
$$

is more clear if we write (1) in an alternative form

$$
i \frac{d A_{j}}{d t}+\gamma\left|A_{j}\right|^{2} A_{j}+\varepsilon\left(A_{j+1}-2 A_{j}+A_{j-1}\right)=0 .
$$


The transformation $A_{j} \rightarrow A_{j} \exp (-2 i t \varepsilon)$ takes (3) into (1). With $\varepsilon=$ $1 /(\Delta x)^{2},(3)$ is seen as a standard finite difference approximation to (2).

The DNLS equation (1) is a special case of a more general equation, the Discrete Self-Trapping (DST) equation ${ }^{34}$

$$
i \frac{d A_{j}}{d t}+\gamma\left|A_{j}\right|^{2} A_{j}+\varepsilon \sum_{k} m_{j k} A_{k}=0 .
$$

Here $M=\left[m_{i j}\right]$ is a $f \times f$ coupling matrix. In physical applications $M$ is real and symmetric, and clearly with $M$ a suitably chosen constant tridiagonal matrix, we can regain (1) or (3). Physically this corresponds to the choice of nearest neighbour couplings. A more general choice for the elements of $M$ introduce longer range couplings or different topologies to the lattice. The distinction between the DST and the DNLS equation is somewhat blurred in modern usage.

One interesting limiting special case of the DST equation is the case of the so-called complete graph model, when

$$
m_{i j}=1-\delta_{i j}
$$

corresponding to a lattice with each point connected directly to every other point on the lattice.

Clearly one can scale $t$ and $\gamma$ in the DNLS model to fix $\varepsilon=1$, and this is often done in the literature. However the more general formulation (1) is useful when one wishes to consider the case $\varepsilon \rightarrow 0$, i.e. the limit of zero coupling, known nowadays as the anti-integrable or anti-continuum limit ${ }^{75}$. In this limit, the solution of (1) is trivial:

$$
A_{j}=\sqrt{\frac{\omega_{j}}{\gamma}} e^{i\left(\omega_{j} t+\alpha_{j}\right)}
$$

where the frequencies $\omega_{j}$ and phases $\alpha_{j}$ can be chosen arbitrarily and independently at each site.

It is worth pointing out to avoid confusion that there are other possible discretizations of the NLS equation, one being the eponymous AblowitzLadik (AL) model ${ }^{3}$

$$
i \frac{d A_{j}}{d t}+\left(1+\frac{1}{2} \gamma\left|A_{j}\right|^{2}\right)\left(A_{j+1}+A_{j-1}\right)=0 .
$$

Another is a model due to Izergin and Korepin ${ }^{52}$ which is rather lengthy to write down, see the book by Faddeev and Takhtajan ${ }^{37}$ for details. Both the AL model and the Izergin-Korepin models have the advantage of being integrable equations ${ }^{2}$, but it can be argued that they are less physically meaningful. The DST equation has been shown to be non-integrable when 
$f>2$ except for the rather unphysical case of a special non-symmetric interaction matrix $M$. It should also be mentioned that an often studied model is the Salerno equation ${ }^{90}$, which contains a parameter interpolating between the AL model (6) with pure inter-site nonlinearity and the DNLS equation (1) with pure on-site nonlinearity. This allows e.g. the use of soliton perturbation theory ${ }^{98,24}$ to elucidate the role of the on-site nonlinearity as a non-integrable perturbation to the integrable AL equation. The Salerno equation was extensively analyzed by Hennig and co-workers ${ }^{50}$ and is also described in the book by Scott ${ }^{92}$.

Another possible source of confusion is that the acronym DNLS is sometimes used for the Derivative Nonlinear Schrödinger equation ${ }^{2}$.

The DST equation (4) can be derived from the Hamiltonian:

$$
H=\sum_{j=1}^{f}\left[\omega_{0}^{(j)}\left|A_{j}\right|^{2}-\frac{\gamma}{2}\left|A_{j}\right|^{4}\right]-\varepsilon \sum_{j \neq k} m_{j k} A_{j}^{*} A_{k}
$$

with canonical variables: $q_{j} \equiv A_{j}$ and $p_{j} \equiv \mathrm{i} A_{j}^{*}$. Here $\omega_{0}^{(j)} \equiv \varepsilon m_{j j}$ are the harmonic frequencies of each uncoupled oscillator ('on-site energies'); with $m_{j k}=\delta_{j, k \pm 1}$ the DNLS equations (1) and (3) are obtained for $\omega_{0}^{(j)}=0$ and $\omega_{0}^{(j)}=2 \varepsilon$, respectively. There is a second conserved quantity, the number (or norm)

$$
N=\sum_{j=1}^{f}\left|A_{j}\right|^{2} .
$$

The integrability of the $f=2$ (dimer) case follows from these two conserved quantities, and in this case all the time-dependent solutions $A_{j}(t), j=1,2$ can be expressed in terms of elliptic functions ${ }^{53}$. One can always scale $A_{j}$ and $\gamma$ so that $N=1$, or alternatively scale $A_{j}$ and $N$ so that $\gamma=1$.

The DNLS Hamiltonian is the starting point for a study of a quantum version of the DNLS system, see the paper by Eilbeck in these proceedings. In particular the quantum analogue of a classical discrete breather can be derived.

There are now almost 300 papers on the DNLS and DST equations, and in this short survey we can only hope to cover a small amount of available material, concentrating on our own interests. Currently a database of papers in this area is held at http://www.ma.hw.ac.uk/ chris/dst. For complementary aspects we recommend the review papers by Hennig and Tsironis $^{50}$ (in particular concerning map approaches with applications to stationary wave transmission) and Kevrekidis et al. ${ }^{63}$ (in particular concerning different types of localized modes and their stability, bifurcation 
and interaction properties), as well as the pedagogical introduction in the textbook by Scott $^{92}$ and the general review of discrete breathers by Flach and Willis ${ }^{43}$.

\section{Stationary Solutions}

Stationary solutions of the DNLS or DST equations are special solutions of the form

$$
A_{j}(t)=\phi_{j} \exp (i \omega t)
$$

where the $\phi_{j}$ are independent of time. Inserting this ansatz into the equations give an algebraic set of equations for the $\phi_{j}$. For example, for DNLS (1), we get

$$
-\omega \phi_{j}+\gamma\left|\phi_{j}\right|^{2} \phi_{j}+\varepsilon\left(\phi_{j+1}+\phi_{j-1}\right)=0
$$

It is this feature that makes the DNLS a relatively simple model to work with. For small periodic lattices up to $f=4$ it is possible to solve the resulting equations exactly and obtain all the families of stationary solutions as a function of $\omega$ and $\gamma$ (for fixed $N$ ), with a fascinating bifurcation structure $^{34}$. The complete graph model can also be solved exactly for any $f^{34}$. For a large or infinite lattice the solutions must be found by numerical methods such as shooting methods or spectral methods. These solutions can then be investigated as a function of the parameters of the equation by numerical continuation methods (see e.g. ${ }^{32}$ for a complete list of solutions for $f=6$ ). If $\gamma$ is sufficiently large, localized solutions are found which decay exponentially for large $|j|$. Two examples are shown in Fig.1. Since these solutions have a periodic time behaviour $\phi_{j} \exp (i \omega t)$, it seems

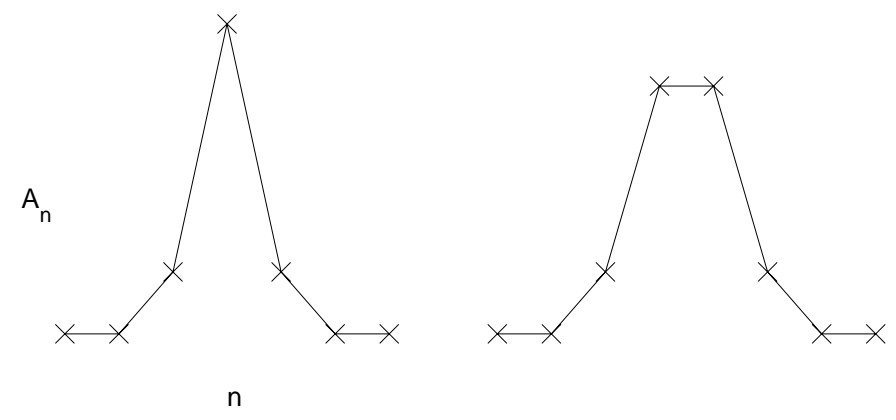

Figure 1. Example: localized stationary solutions of the DNLS model 
appropriate to call them "breather" solutions. Another motivation is that the DNLS equation can be derived from the discrete Klein-Gordon equation, describing a lattice of coupled anharmonic oscillators, via a multiscale expansion in the limits of small-amplitude oscillations and weak inter-site coupling ${ }^{68,28,83}$. The discrete breathers of this lattice are then represented as stationary solutions to the DNLS equation. The reader should note that in the early days of DNLS studies, when breathers in discrete systems were not so well understood, these solutions were often called solitons.

The stability of such solutions in time can be investigated by looking at general perturbations in the rotating frame of the solutions ${ }^{18}$

$$
\tilde{A}_{j}(t)=\left(\phi_{j}+\delta u_{j}(t)\right) \exp (i \omega t)
$$

This reduces the linear stability problem to a study of a linear eigenvalue problem. It is perhaps to be expected that the stability of a branch of stationary solutions can change at a bifurcation point. What is surprising is that, since the eigenvalue problem is not self-adjoint, solutions can also change stability at other points on the branch. Usually the single-site peaked ('site-centred') solution shown at the 1.h.s. of Fig.1 turns out to be stable, whereas the two-site peaked ('bond-centred') solution shown on the r.h.s. is not.

For the case of an infinite lattice, both solutions in Fig.1 can be smoothly continued versus coupling $\varepsilon$ (or, equivalently by rescaling, versus $\omega$ ) for all $\varepsilon$, without encountering any bifurcations. For $\varepsilon \rightarrow 0$, the site-centred solution will be completely localized at the central site with all other oscillator amplitudes zero, while the bond-centred solution becomes completely localized on the two central sites. For $\varepsilon \rightarrow \infty$ both solutions are smoothly transformed into the same soliton solution of the continuous NLS (2) (which explains why they are sometimes also termed 'discrete solitons'). As there are no bifurcations, the site-centred solution is stable and the bond-centred unstable for all $\varepsilon$ in the infinite chain. Comparing the value of the Hamiltonian ('energy') of the two solutions for a fixed $N$, one finds that the site-centred solution always has the lowest energy. This energy difference has been proposed to act as a sort of Peierls-Nabarro potential barrier ${ }^{65}$. Another property of these two solutions in infinite 1D lattices is that they exist for arbitrarily small $\gamma$ (or arbitrarily small $N$ for fixed $\gamma$ )

Historical note. Although the stationary DNLS equation (10) was derived already by Holstein in 1959 in his pioneering work on polarons in molecular crystals ${ }^{51}$, the first systematic study of its single-peak breather solution as an exact solution to the fully discrete equations was performed by Scott and MacNeil in $1983^{94}$, following Scott's interest in Davydov 
solitons on proteins. They investigated the family of single-peak stationary solutions using shooting methods running on a Hewlett-Packard programmable calculator. Further interest in Davydov solitons on protein molecules led to a study of a related molecule called acetanilide. A model of the crystalline state of this molecule was set up which was essentially four coupled DNLS systems. Techniques to map out the families of stationary solutions on this system were developed, including path-following from the anharmonic limit ${ }^{33}$. It was then realised that the single DNLS system was of independent interest, which led to the work described in ${ }^{34}$.

Later and independently, Aubry, MacKay and co-workers developed a much more general approach along these lines to the breather problem in arbitrary systems of coupled oscillators ${ }^{75,11}$. In this context, much new attention was directed to the DNLS model and its stationary solutions. In addition, two more large bursts of interest into studies of the DNLS equation have appeared recently, following the experimental progress in the fields of nonlinear optical waveguide arrays ${ }^{36}$ and Bose-Einstein condensates trapped in periodic potentials arising from optical standing waves ${ }^{8}$. These applications will be discussed briefly below. Since the DNLS equation is of general applicability and appears in completely different physical fields, new researchers drawn to its study have not always been aware of earlier results. Thus many of its properties have been independently rediscovered and appeared several times in the literature in different contexts during the last two decades.

\section{Disorder}

One natural generalization to the DNLS equation (1) or (3) is to consider non-constant coupling parameters $\varepsilon_{j k}$, equivalent to nontrivial distributions of the elements $m_{j k}$ of the matrix $M$ in the DST equation (4). One may also consider site dependent $\gamma_{j}$ as well. An early application, in the large $f$ case, was to model the dynamics of the energy distribution of modes on a globular protein. Feddersen ${ }^{38}$ considered interactions among $\mathrm{CO}$ stretch oscillations in adenylate kinase, which comprises 194 amino acids $(f=194)$. Since the structure of this enzyme has been determined by x-ray analysis, the $f(f-1) / 2=18721$ off-diagonal elements of the dispersion matrix $M$ were calculated from Maxwell's equations. Also diagonal elements were selected from a random distribution, and the degree of localization of a particular stationary solution of the form (9) with real $\phi$ was defined by evaluating the quotient $\sum \phi_{j}^{4} / \sum \phi_{j}^{2}$.

This numerical study revealed two features. Firstly, at experimentally reasonable levels of nonlinearity $(\gamma)$, stable localized solutions were ob- 
served near some but not all of the lattice sites. Secondly, this anharmonic localization was observed to be distinctly different from "Anderson localization", a property of randomly interacting linear systems ${ }^{9}$. Thus none of the stationary states that were observed to be highly localized at large $\gamma$ remained so as $\gamma$ was made small. Also, none of the states that were localized at $\gamma=0$ (i.e., Anderson localized) remained so as $\gamma$ was increased to a physically reasonable level.

The transition between Anderson localized modes and breather states has more recently been extensively analysed in a series of papers by Kopidakis and Aubry for general coupled oscillator chains ${ }^{69,71,70}$ (see also Archilla et al. ${ }^{10}$ for a slightly different model), and has to its larger parts been understood. The generic scenario, valid also for the DNLS model, is consistent with Feddersen's observations but too complicated to describe in detail here. Briefly, there are two kinds of localized breather solutions in a disordered nonlinear lattice: 'extraband discrete breathers' (EDBs) with frequencies outside the spectrum of linear Anderson modes, and 'intraband discrete breathers' (IDBs) with frequencies inside the linear spectrum. EDBs cannot be smoothly continued versus frequency into IDBs but are lost in cascades of bifurcations. IDBs on the other hand can be continued outside the linear spectrum, but not into EDBs but only into a certain type of spatially extended multi-site breathers. The IDBs can only exist as localized solutions inside the linear spectrum provided their frequencies do not resonate with linear Anderson modes. However, for an infinite system the linear spectrum becomes dense so that the allowed frequencies for localized IDBs must constitute a (fat, i.e. of non-zero measure) Cantor set! In fact, for the DNLS case the latter result had been rigorously obtained already in 1988 by Albanese and Fröhlich ${ }^{5}$; see also ${ }^{45,6}$ for other early mathematical results on the DNLS model with disorder.

It is interesting to remark that the general scenario with two types of discrete breathers, EDBs and IDBs, where the latter exist as localized single-peaked solutions only in-between resonances with linear modes, is not peculiar for random systems, but observed also in other situations when the linear spectrum is discrete with localized eigenmodes. A very recently studied example ${ }^{85}$ is the DNLS model (1) with an added linearly varying on-site potential $\omega_{0}^{(j)}=\alpha j$. In this case the linear spectrum constitutes a so-called Wannier-Stark ladder (WSL) of equally spaced eigenfrequencies, with eigenstates localized around each lattice site, giving rise to Bloch oscillations (recently experimentally observed in waveguide arrays ${ }^{82,84}$ ). Then, resonances were shown to result in 'hybrid discrete solitons', interpreted as bound states of single-peaked IDBs and satellite tails corresponding to 
nonlinearly modified Wannier-Stark states localized some distance away from the main peak. Due to the finite (constant) distance between the linear eigenfrequencies in the WSL, IDBs remain single-peaked in frequency intervals of finite length, in contrast to the IDBs in random systems.

The fact that nonlinearity modifies localized linear modes into extended nonlinear solutions should be of some physical importance, since it provides a mechanism for transport in random systems. In fact, this aspect was considered also by Shepelyansky in $1993^{95}$, who used the well-known Chirikov criterion of overlapping resonances to argue that, above some critical nonlinearity strength $\gamma_{c}$, the number of excited linear modes (and thus the spatial width) for a typical initially localized excitation in the DNLS model would spread sub-diffusively as $(\Delta n)^{2} \sim t^{2 / 5}$ (for linear random systems, $(\Delta n)^{2}$ remains finite under very general conditions).

We also mention that the case with disorder residing purely in the nonlinearity strengths $\gamma_{j}$ was studied by Molina and Tsironis ${ }^{79}$. In this case only partial localization of an initially single-site localized excitation could be found for large nonlinearities (dynamical self-trapping, corresponding to asymptotic approach to an exact discrete breather), while some portion was found to always escape ballistically (i.e. spreading as $(\Delta n)^{2} \sim t^{2}$ ) leading to absence of complete localization. The scenario with partial self-trapping above a critical nonlinearity strength combined with asymptotic spreading through small-amplitude waves appears very generally for single-site initial conditions in the DNLS model, with or without disorder ${ }^{78,15,58}$.

\section{Mobile breathers}

Since the DNLS equation is an approximation to the equations describing Davydov solitons, which are thought from numerical studies to be mobile, it is natural to ask whether the sort of breathers shown in Fig.1 can move if sufficiently perturbed. The first attempt to model this was made in a relatively obscure conference proceedings ${ }^{31}$ in 1986 . The key to getting mobility is to realise that a shape like Fig. 1 will move if the figure represents $\left|A_{j}\right|^{2}$ but the phase is no longer constant and rotates through $2 \pi$ as we traverse the breather. The same paper reported a very preliminary study of the interaction of the moving breather with an "impurity", or more precisely a long-range interaction due to the curved nature of the chain. There is now a growing literature on trapping of mobile breathers due to curved chains and long range-coupling (c.f. ${ }^{38,46}$ ) and on trapping due to local impurities in the lattice (c.f. ${ }^{49}$ ). Regrettably, due to space considerations, we have omitted any further discussion of this interesting area.

Feddersen ${ }^{39,30}$ used spectral and path-following methods to make a more 
detailed numerical study of travelling breathers in the DNLS system using the ansatz

$$
A_{j}(t)=u(z) \exp \{i(\omega t-\kappa j)\}, \quad z=j-c t .
$$

Note that $c \neq \omega / \kappa$, i.e. the solution is regarded as a solitary pulse modulated by a carrier wave moving at a different velocity. His studies show a solution with this form to a high degree of numerical accuracy for a range of parameter values. However this numerical evidence cannot be regarded as a rigorous proof for the existence of moving breathers in the DNLS system, and this is still an outstanding question.

Much recent attention has been drawn to mobile breathers in general oscillator chains (see several other contributions to these proceedings), and many of these results can be transferred also to DNLS chains. Here, we wish to just mention particularly some results of Flach et al. ${ }^{44}$ (see also ${ }^{41}$ ) who used an inverse approach, choosing particular given profiles of travelling waves and finding equations of motion having these as exact solutions. Generalizing the DNLS equation (1) by replacing $\gamma\left|A_{j}\right|^{2}$ with $G\left(\left|A_{j}\right|^{2}\right)$ and $\varepsilon$ with $\varepsilon+F\left(\left|A_{j}\right|^{2}\right)$, where $F$ and $G$ are functions to be determined, and choosing $A_{j}$ of the form (11) with real $u$, they could determine explicit expressions for the functions $F$ and $G$ for which the particular solution $A_{j}$ exists as an exact travelling wave. In this way, they could e.g. reproduce the AL-model (6) for $G \equiv 0$ by choosing $A_{j}$ to be its well-known soliton solution. Moreover, they could prove that no such travelling solution with pulse shaped $u$ could exist for a pure on-site nonlinearity $(F \equiv 0)$, and thus not for the DNLS equation (1). However, this does not prove the absence of exact moving localized DNLS breathers, for (at least) two reasons. (i) The envelope $u(z)$ could contain a non-trivial space-dependent complex phase not absorbable into the $\exp \{-i \kappa j\}$ factor. In fact, the solutions numerically found by Feddersen contained such a phase. (ii) Moving breathers could have a time-varying (e.g. periodic) shape function $u(z, t)$. This could be possible since the stationary DNLS breathers, in the regime where mobility is numerically observed, exhibit internal shape modes ('breathing modes') which can be found as localized time-periodic solutions to the linearized equations $^{57,67,56,63}$.

\section{Chaotic Solutions}

Since the DNLS equation for $f>2$ is not integrable, it might be expected that it has solutions exhibiting Hamiltonian chaos, and in fact the first study of the DST equation showed chaotic-looking trajectories in the $f=3$ case $^{34}$. A more thorough analysis of this case was carried 
out by Cruzeiro-Hansson et al. ${ }^{25}$, who estimated the region of both classical and quantum phase space occupied by chaotic states. A number of other studies have been carried out since then. For example, Hennig and coworkers $^{48}$ considered a DST trimer (4) with $m_{11}=m_{22}=m_{33}=0$ and $m_{13}=m_{23} \ll m_{12}=m_{21}$, i.e. an (integrable) dimer interacting weakly with the third oscillator. Then, a Melnikov approach could be used to show the existence of homoclinic chaos. A similar approach for the case $f=4$ with a dimer interacting weakly with the two other sites also demonstrated the presence of Arnold diffusion ${ }^{47}$. In the opposite limit of large $f$, homoclinic chaos has also been demonstrated and analyzed through a Melnikov analysis of a perturbed continuous NLS equation ${ }^{17}$.

As another example of chaotic behaviour, Eilbeck ${ }^{32,35}$ showed that on a $f=6$ periodic lattice modelling benzene, a mobile breather could propagate which hopped around the lattice in a random way, even reversing its direction of motion at unpredictable intervals.

\section{2-dimensional DNLS lattices}

As follows from the general theory of MacKay and Aubry ${ }^{75}$, breathers exist also in higher dimensions. While we are aware of very few explicit results for the DNLS model in three dimensions ${ }^{42}$, the two-dimensional case has been rather thoroughly studied. Some recent results are described in ${ }^{63}$. Instead of attempting to give a complete survey here, we will concentrate on discussing the main differences to the one-dimensional case.

In the $2 \mathrm{D}$ case and for a square lattice, the DNLS equation (3) with $\gamma=\varepsilon=1$ is readily generalized to

$i \frac{d A_{m, n}}{d t}+\left|A_{m, n}\right|^{2} A_{m, n}+A_{m+1, n}+A_{m-1, n}+A_{m, n+1}+A_{m, n-1}-4 A_{m, n}=0$,

and stationary solutions of the form (9) with frequency $\omega$ can be found analogously to the 1D case. The single-site peaked discrete soliton (breather) was first thoroughly studied in ${ }^{77,72}$. The following characteristics should be mentioned: (i) The solution can be smoothly continued from a single-site solution at the anti-continuum (large-amplitude) limit $\omega \rightarrow \infty$ to the so called ground state solution of the continuous 2D NLS equation ${ }^{87}$ in the small-amplitude limit $\omega \rightarrow 0$. (ii) There is an instability-threshold at $\omega \sim 1$, so that the solution is stable for larger $\omega$ ('discrete branch') and unstable for smaller $\omega$ ('continuum-like branch'). (iii) The stability change is characterized by a change of slope in the dependence $N(\omega)$, so that $\frac{d N}{d \omega}>0$ $(<0)$ on the stable (unstable) branch. (A similar criterion exists also for single-site peaked solutions to the $1 \mathrm{D}$ DNLS equation with on-site nonlin- 
earities of arbitrary power ${ }^{73}$.) (iv) The value of the excitation number $N$ at the minimum is nonzero, and thus there is an excitation threshold ${ }^{99}$ for its creation, in contrast to the $1 \mathrm{D}$ case (3) where $N \rightarrow 0$ as $\omega \rightarrow 0$ for fixed $\gamma=\epsilon=1$. A similar scenario occurs also in $3 \mathrm{D}^{42}$. The effect of this excitation threshold in 2D was recently proposed to be experimentally observable in terms of a delocalizing transition of Bose-Einstein condensates in optical lattices $^{61}$. (v) The dynamics resulting from the instability on the unstable branch is, in the initial stage, similar to the collapse of the unstable ground state solution of the continuous 2D NLS equation ${ }^{87}$, with increased localization and blow-up of the central peak. In contrast to the continuum case, however, this process must be interrupted since the peak amplitude must remain finite, and the result is a highly localized 'pulson' state where the peak intensity $\left|A_{m, n}\right|^{2}$ oscillates between the central site and its four nearest neighbours ${ }^{22}$. This process has been termed 'quasicollapse'. ${ }^{73,77}$ It is not known whether these pulson states represent true quasiperiodic solutions to the DNLS equation (see below).

As was shown by MacKay and Aubry ${ }^{75,11}$ under very general conditions, two-dimensional lattices allow for a new type of localized solutions, 'vortex-breathers', with no counterpart in 1D. They can be constructed as multi-site breathers by continuation from an anti-continuum limit of a cluster of single-site breathers with identical frequencies but with uniformly spatially varying phases constituting a closed loop, such that the total phase variation around the loop ('topological charge') is a multiple of $2 \pi$. The simplest examples are three breathers in a triangle phase shifted by $2 \pi / 3$, or four breathers in a square phase shifted by $\pi / 2$. The general existence and stability theorems ${ }^{75,11}$ (valid also for the DNLS equation) guarantee that such solutions exist as localized solutions for weak enough coupling, and that certain configurations are linearly stable. As a consequence of the phase torsion, such solutions will carry a localized circulating current when the coupling is nonzero. For the DNLS model, vortex-breathers for a square 2D lattice were first obtained in ${ }^{57}$. Typically they become unstable as the coupling is increased; the mechanisms of these instabilities were described in some detail in ${ }^{76,63}$.

Let us finally also mention a recent study ${ }^{62}$ exploring numerically different types of breathers (including vortex-breathers) and their stability in triangular and hexagonal DNLS-lattices. 


\section{Quasiperiodic Breathers}

A particular feature of the DNLS equation, distinguishing it from generic anharmonic lattice models, is the existence of continuous families of exact, spatially localized solutions of the form (9) but where the amplitudes $\phi_{j}$ in the rotating frame are not time-independent but time-periodic (with nonharmonic time-dependence). Such solutions are obtained by adding a term $i \dot{\phi}_{j}$ to the left-hand side of Eq.(10). Thus, these solutions are in general quasiperiodic with two incommensurate frequencies in the original amplitudes $A_{j}$ (although they may also be periodic if the frequency relation is rational). At first, one may not be surprised by the existence of quasiperiodic solutions, since at least for finite-size lattices they should appear as KAM tori. However, generically (i) one would not expect them to appear in continuous families since they should be destroyed for rational frequency relations; and (ii) one would not expect them to survive as localized solutions in infinite lattices since the presence of two incommensurate frequencies in a generic anharmonic system would generate all possible linear combinations of the frequencies, i.e. a dense spectrum implying that resonance with the continuous spectrum should be unavoidable, and the breather should radiate and decay.

The key point to realize why, in spite of this, quasiperiodic breathers with two incommensurate frequencies do exist in the DNLS lattice is to note that the first frequency $\omega$ in (9) always yields harmonic oscillations, and thus no multiples of this frequency are generated. The origin to this is the phase invariance of the DNLS equation, i.e. invariance under transformations $A_{j} \rightarrow A_{j} e^{i \alpha}$, related to the norm conservation law (8) by Noether's theorem. A recent result ${ }^{13}$ proves that very generally, each conservation law in addition to the Hamiltonian yields possibility for existence of quasiperiodic breathers with one additional frequency.

The existence of quasiperiodic DNLS-breathers in infinite lattices was first proposed by MacKay and Aubry ${ }^{75}$, and later explicit proofs of existence and stability as well as numerical demonstrations for some particular examples were given ${ }^{55,57}$ (earlier findings of quasiperiodic solutions in DNLS-related models had concerned mainly small systems ${ }^{53,7}$ or the integrable AL model $\left.{ }^{16}\right)$. As some renewed interest has appeared on this topic ${ }^{63}$, it is useful to comment on the differences between these two approaches. The solutions in ${ }^{55,57}$ (see also ${ }^{13}$ ) were constructed as multi-site breathers by continuation from the anti-continuum limit $\varepsilon=0$ of solutions with two (or more) sites oscillating with non-zero amplitude according to (5) with two different (generally incommensurate) frequencies $\omega_{1}$ and $\omega_{2}$. Except 
for some particular relations between the frequencies where resonances appear, such solutions can always be continued to some non-zero $\varepsilon$. On the other hand, the solutions discussed in ${ }^{63}$ originated in internal-mode excitations (i.e. time-periodic localized solutions to the linearized equations) of a particular stationary solution, the so-called 'twisted localized mode ${ }^{, 27}$ (TLM). As for the bond-centred breather in Fig.1, the anti-continuum version of this solution has two neighbouring sites oscillating with equal $\left|A_{j}\right|^{2}$; however for the TLM these sites are oscillating in anti-phase so that the solution is spatially antisymmetric. This solution exists and is linearly stable for small $\varepsilon .{ }^{55}$ Now, the occurrence of linear internal-mode oscillations is a very common phenomenon ${ }^{67}$. However, in most cases such oscillations do not yield true quasiperiodic solutions of the fully nonlinear equations since typically some harmonic will resonate with the linear continuous phonon spectrum, implying that these oscillations decay in time. This scenario appears e.g. for the single-site peaked DNLS-breather ${ }^{56}$. The interesting discovery by Kevrekidis and co-workers was, that for the particular case of the TLM, there are certain intervals in $\varepsilon$ where all higher harmonics of the internal mode frequency are outside the continuous spectrum, and thus in these intervals the oscillating solutions of the linearized equations could be continued into truly quasiperiodic localized solutions of the nonlinear equation. As the allowed intervals are away from $\varepsilon=0$, it is clear that this approach yields solutions which could not be obtained by direct continuation from the anti-continuum limit.

\section{Wave Instabilities}

Another important class of solutions in anharmonic lattices are space-time periodic travelling waves. For the DNLS model such solutions are very simple, since they are just rotating-wave solutions of the type (11) with constant $u=|A|$. Direct substitution into the DNLS equation (using the form (3)) yields the nonlinear dispersion relation

$$
\omega=-4 \varepsilon \sin ^{2} \frac{\kappa}{2}+\gamma|A|^{2} .
$$

Linear stability analysis shows ${ }^{18,68}$ that the travelling waves are stable if and only if $\frac{\gamma}{\epsilon} \cos \kappa<0$. Thus, for $\frac{\gamma}{\epsilon}>0$ only waves with $\pi / 2<|\kappa| \leq \pi$ are stable, while waves with small wave vectors $0 \leq|\kappa| \leq \pi / 2$ are unstable through a modulational instability analogously to the continuous NLS equation. This instability destroys the homogeneous amplitude distribution of the wave, and typically ${ }^{28,88,89}$ results in the creation of a number of smallamplitude mobile localized excitations ('breathers'), which through interaction processes (see below) may coalesce into a small number of standing 
large-amplitude breathers. Thus, the plane-wave modulational instability was proposed ${ }^{28}$ generally to constitute the first step towards energy localization in nonlinear lattices (including DNLS).

Now, in a linear system one may always take linear combinations of counter-propagating waves $e^{ \pm i \kappa j}$ to obtain standing waves (SWs) of the form $\cos (\kappa j+\beta)$. The same is of course not true in a nonlinear system due to lack of superposition principle; still however there generally exist nonlinear continuations of the linear standing waves, although they cannot be written as superpositions of counter-propagating travelling waves. Such nonlinear standing waves were investigated in detail for general coupled oscillator chains in ${ }^{83}$ (the results for the DNLS chain were more concisely summarized in ${ }^{60}$ ). Without going into too much detail, let us state some main conclusions, referring to the DNLS form (3) with $\gamma=1$. (i) SWs with given wave vector $\kappa$ exist as stationary solutions of the form (9) for all values of $\frac{\omega}{\varepsilon}>-4 \sin ^{2} \frac{\kappa}{2}$. In the lower limit (corresponding to the dispersion relation (13) for a linear wave), the wave is a linear standing wave. (ii) In the anti-continuum limit $\frac{\omega}{\epsilon} \rightarrow \infty$ a SW with wave vector $\kappa$ is described by a particular spatially periodic (or quasiperiodic if $\kappa$ is irrational) repetition of local on-site solutions of the form (5) of oscillating and zero-amplitude solutions. The oscillating sites have the same frequency $\omega$ but generally alternating phases $\alpha=0, \pi$. (iii) For each wave vector $\kappa$ there are only two different distinct (modulo lattice translations) SW families corresponding to different spatial phases $\beta$ of the linear $\mathrm{SW} \cos (\kappa j+\beta)$. They appear as hyperbolic respectively elliptic periodic points in the map defined by the stationary DNLS equation (10). (iv) One of the SW families is stable in a regime of large $\frac{\omega}{\epsilon}$, while close to the linear limit all nonlinear $S W s$ with $\kappa \neq \pi$ are unstable for infinite systems! The instability for the 'most stable' waves is of oscillatory type (i.e. corresponding to complex eigenvalues of the linear stability eigenvalue problem).

Investigating the long-time dynamics resulting from the SW instabilities, completely different scenarios were found ${ }^{60}$ for $|\kappa|<\pi / 2$ and $|\kappa|>\pi / 2$, respectively. For the first case one finds after long times persisting large-amplitude standing breathers, while for the second case a 'normal' thermalized state is obtained. In fact, this division of the available phase space into two isolated regimes of qualitatively different asymptotic dynamics was first found by Rasmussen et al. ${ }^{89}$, and shown to correspond to a phase transition through a discontinuity in the partition function in the Gibbsian formalism. In terms of the Hamiltonian and norm densities for a chain of $f$ sites, the phase transition line was obtained as $\frac{H}{f}=-\gamma\left(\frac{N}{f}\right)^{2}$, which can be seen to correspond exactly to a SW with wave 
vector $|\kappa|=\pi / 2$. Note that the existence of the second conserved quantity $N$, which is peculiar for DNLS-type models, is crucial in this context.

Another interesting observation is that taking the limit $\kappa \rightarrow \pi$ for one of the nonlinear SWs generated from the anti-continuum limit as above, one obtains a solution consisting of a stable background wave with $\kappa=\pi$ having a single defect site of zero-amplitude oscillation inserted into it. This solution can be smoothly continued to the continuum limit, where it is seen to correspond to the dark-soliton solution of the defocusing NLS equation (note that the transformation $A_{j}=(-1)^{j} A_{j}$ in (1) is equivalent to reversing the sign of $\left.\frac{\gamma}{\varepsilon}\right)$. Also the discrete dark soliton ('dark breather') has been shown to be stable close to the anti-continuum limit, but unstable through an oscillatory instability close to the continuum limit for arbitrarily weak discreteness ${ }^{59}$. The typical outcome of this instability is a spontaneous motion $^{66}$. As for the case of ordinary moving breathers, it is still an open question whether moving dark breathers exist as exact solutions, and current research is devoted to this issue. However, numerical evidence that they can exist at least to a very high numerical accuracy was given in the work of Feddersen ${ }^{39,40}$.

Let us also mention that asymmetric discrete dark solitons, with different left and right background amplitudes, can exist as quasiperiodic solutions of the type described in the previous section. Such solutions were analyzed by Darmanyan et $\mathrm{al}^{26}$ and are subject to similar instabilities.

\section{Breather Interactions}

In general, one cannot conclude from a linear stability analysis that a solution is fully stable, but only that small perturbations at least cannot grow exponentially in time. However, for the single-peaked DNLS-breather, a stronger result is obtained ${ }^{99}$ : such solutions are orbitally Lyapunov stable for norm-conserving perturbations. This basically means that small breather perturbations will remain small (modulo a possible phase drift) for all times. This result is a consequence of the single-site breather being a ground state solution, in the sense that among all possible solutions at a given norm, it has the smallest value of the Hamiltonian. Thus, once again we find a property where norm conservation is crucial, and thus one should not expect that Lyapunov stability is a generic property of breathers in Hamiltonian lattices.

Still there are important issues to address concerning the fate of perturbed breathers, which cannot be predicted from stability theorems. One issue is breather-breather interactions, which correspond to large perturba- 
tions. Some preliminary work was done by Feddersen ${ }^{39}$, who showed that the collison of two breathers of equal amplitude travelling in opposite directions was close to elastic. In the more general case the situation is more complicated.

Accumulated knowledge from several numerical experiments on general breather-carrying systems, in particular by Peyrard and coworkers (e.g. ${ }^{86}$ ) has lead to the conjecture, that in collisions between standing largeamplitude breathers and moving small-amplitude breathers, big breathers systematically grow at expense of the small ones. For the DNLS model, such a scenario was described in 88 .

Another interesting issue is breather interactions with small-amplitude phonons, where also the long-time dynamics cannot be predicted from stability theorems since extended phonons in infinite lattices have infinite norm. A first approach ${ }^{74,64}$ is to consider this as a linear scattering problem, with incoming, outgoing and reflected linear phonons scattered by the breather. Then, within the linear framework, one finds the scattering on a single-peaked DNLS-breather to be always elastic. In certain cases, even perfect transmission or perfect reflection of phonons appear ${ }^{74,64}$.

However, going beyond linear theory the scattering process is generally inelastic, and the breather may absorb or emit energy to the surrounding phonons. These processes were investigated in ${ }^{56,54}$ using a multiscale perturbational approach. It was found ${ }^{56}$, that under certain conditions a breather can pump energy from a single phonon and continuously grow with a linear growth rate. This process is always associated with generation of second-harmonic outgoing phonon radiation. On the other hand, it was also found ${ }^{54}$ that breather decay could only happen if two or more different phonons were initially simultaneously present. An additional interesting observation $^{54}$ was that beyond a certain breather amplitude $\left(\left|A_{0}\right|^{2} \gtrsim 5.65\right.$ corresponding to $\omega>4 \epsilon$ for the DNLS of form (3) with $\gamma=1$ ), all lowestorder growth and decay processes disappear. Thus, this explains why, once created, breathers with large amplitude are extremely stable also for nonnorm-conserving perturbations.

Let us finally mention also some results obtained ${ }^{21}$ for an extended DNLS model, which has very recently received renewed attention in the description of ultrafast catalytic electron transfer ${ }^{12}$. To model the interaction of an electron, or exciton, with a classical phonon system treated as a thermal bath, the DNLS equation is appended with the terms $\left[-\eta \frac{d}{d t}\left(\left|\psi_{j}\right|^{2}\right)+h_{j}(t)\right] \psi_{j}$, where the first term is a nonlinear damping term providing dissipation, and the second term is a fluctuation term which as a crudest approximation is taken as a Gaussian white noise. This ex- 
tended DNLS equation conserves excitation number but not the Hamiltonian. Then, it was shown ${ }^{21}$ that breathers are always ultimately destroyed, but that strongly localized breathers may be very long-lived for weak noise. The decay was shown to be linear in time, with decay rate proportional to $D(\varepsilon / \gamma)^{2}$, where $D$ is the noise variance (here $N=1$ is assumed). It would be highly interesting to know whether similar behaviour could appear also in more realistic models with coloured noise, since the white noise can be considered to be somewhat unphysical having infinite frequency content.

\section{Applications}

We have already mentioned the Holstein polaron model as (to our knowledge) the first ${ }^{51}$ suggested application of a DNLS equation. Likewise, we mentioned Davydov solitons ${ }^{29,92,91}$. Another early motivation for the study of the DNLS/DST equation was within the theory of Local Modes of small molecules $^{93}$. The two latter topics are well described in the textbook by Scott $^{92}$. Here we just briefly discuss the two applications which have attracted the most attention during the last five years, namely coupled optical wave guides and Bose-Einstein condensates (BEC).

The modelling of two coupled optical waveguides, interacting through a nonlinear material, by a DNLS dimer equation was suggested already in 1982 by Jensen ${ }^{53}$. Later work $^{23}$ extended these ideas and proposed the DNLS equation to describe discrete self-focusing in arrays of coupled waveguides. Many works followed proposing the applicability of different properties of the DNLS equation for nonlinear optical purposes; here we just mention the investigation of packing, steering and collision properties of self-localized beams ${ }^{4}$, and the use of discreteness effects to obtain a controlled switching between different guides in the array ${ }^{14}$. The success of the DNLS equation in describing discrete spatial solitons in waveguide arrays was first experimentally confirmed in $1998 .{ }^{36}$ Later experimental work showed the existence of propagating discrete solitons and confirmed the DNLS predictions of a Peierls-Nabarro barrier ${ }^{81}$ as well as that of nonlinear Bloch oscillations ${ }^{82}$. More recently, also dark discrete solitons were observed $^{80}$.

In the context of Bose-Einstein physics, the use of the dimer DNLS equation was (to our knowledge) first suggested by Smerzi et al. ${ }^{96}$ to model two weakly coupled BEC in a double-well trap. Later ${ }^{97}$, the full DNLS equation was proposed to model the earlier quoted experiment ${ }^{8}$ with a BEC trapped in a periodic potential, and the existence of discrete solitons/breathers for such experiments was predicted. A large amount of theoretical predictions, based on DNLS dynamics, for different phenomena to occur in BEC arrays 
has appeared in the last year, of which we here, quite randomly, just quote 1,61 . So far, most of the predictions are awaiting experimental verification. Some experimental confirmation that, at least to some extent, BEC in periodic potentials can be treated with DNLS models, under the condition that the inter-well potential is much larger than the chemical potential, has appeared very recently ${ }^{19,20}$. In these experiments the BEC was trapped in an optical lattice superimposed on a harmonic magnetic potential, and modelled by a DNLS equation with an additional quadratic on-site term $\Omega j^{2} A_{j}$. The observed frequency of the Josephson-like coherent oscillations of the BEC centre-of-mass in the magnetic trap was shown to agree with DNLS predictions ${ }^{19}$. Moreover, changing the centre of the magnetic potential led to a transition from the (superfluid) regime of coherent oscillations into an insulator regime with the condensate pinned around the potential centre $^{20}$. The onset of the transition was interpreted as the result of a discrete modulational instability, and could be estimated from the DNLS model. Many new experiments in this exciting field are awaited in the near future!

\section{Conclusions}

We hope the reader has enjoyed this brief introduction to this fascinating topic. We are conscious of the many details, figures and areas that we have left out, either because of space restrictions or because the topics are covered in depth elsewhere. To do the subject full justice would require a whole volume.

\section{Acknowledgements}

We thank all our colleagues, too many to mention explicitly, which in one way or another have contributed to this field. Special thanks are due to Sergej Flach and Thomas Pertsch for their helpful assistance, and to Rolf Riklund and Michael Öster for reading the manuscript. JCE would like to thank the EU for the financial support of the LOCNET programme. MJ would like to thank the Swedish Research Council for support.

\section{References}

1. F.Kh. Abdullaev, B.B. Baizakov, S.A. Darmanyan, V.V. Konotop, and M. Salerno. Nonlinear excitations in arrays of Bose-Einstein condensates. Phys. Rev. A, 64:043606-1-10, 2001.

2. M. J. Ablowitz and P. A. Clarkson. Solitons, nonlinear evolution equations, and inverse scattering. CUP, Cambridge, 1991. 
3. M. J. Ablowitz and J. F. Ladik. A nonlinear difference scheme and inverse scattering. Stud. Appl. Math., 55:213-229, 1976.

4. A. B. Aceves, C. Deangelis, T. Peschel, R. Muschall, F. Lederer, S. Trillo, and S. Wabnitz. Discrete self-trapping, soliton-interactions, and beam-steering in nonlinear wave-guide arrays. Phys. Rev. E, 53:1172-1189, 1996.

5. C. Albanese and J. Fröhlich. Periodic solutions of some infinite-dimensional Hamiltonian systems associated with non-linear partial difference equations I. Commun. Math. Phys., 116:475-502, 1988.

6. C. Albanese and J. Fröhlich. Periodic solutions of some infinite-dimensional Hamiltonian systems associated with non-linear partial difference equations. II. Commun. Math. Phys., 119:677-699, 1988.

7. J. D. Andersen and V. M. Kenkre. Exact solutions for the quantum nonlinear trimer. Phys. Stat. Sol. B, 177:397-404, 1993.

8. B.P. Anderson and M.A. Kasevich. Macroscopic quantum interference from atomic tunnel arrays. Science, 282:1686-1689, 1998.

9. P. W. Anderson. Local moments and localized states. Rev. Mod. Phys., 50:1191-201, 1978.

10. J. F. R. Archilla, R. S. MacKay, and J. L. Marín. Discrete breathers and Anderson localization: two faces of the same phenomenon? Physica D, 134:406418, 1999 .

11. S. Aubry. Breathers in nonlinear lattices: existence, linear stability and quantization. Physica D, 103:201-250, 1997.

12. S. Aubry and G. Kopidakis. A nonlinear dynamical model for ultrafast catalytic transfer of electrons at zero temperature. arXiv:cond-mat/0210215, 2002.

13. D. Bambusi and D. Vella. Quasi periodic breathers in Hamiltonian lattices with symmetries. Disc. Cont. Dyn. Syst., 2:389-399, 2002.

14. O. Bang and P.D. Miller. Exploiting discreteness for switching in waveguide arrays. Opt. Lett., 21:1105-1107, 1996.

15. L. J. Bernstein, K. W. Delong, and N. Finlayson. Self-trapping transitions in a discrete NLS model with localized initial conditions. Phys. Letts. A, 181:135-141, 1993.

16. D. Cai, A.R. Bishop, and N. Grønbech-Jensen. Spatially localized, temporally quasiperiodic, discrete nonlinear excitations. Phys.Rev.E, 52:R5784-R5787, 1995.

17. A. Calini, N.M. Ercolani, D.W. McLaughlin, and C.M. Schober. Mel'nikov analysis of numerically induced chaos in the nonlinear Schrödinger equation. Physica D, 89:227-260, 1996.

18. J Carr and J C Eilbeck. Stability of stationary solutions of the discrete selftrapping equation. Phys. Letts. A, 109:201-204, 1985.

19. F.S. Cataliotti, S. Burger, C. Fort, P. Maddaloni, F. Minardi, A. Trombettoni, A. Smerzi, and M. Inguscio. Josephson junction arrays with BoseEinstein condensates. Science, 293:843-846, 2001.

20. F.S. Cataliotti, L. Fallani, F. Ferlaino, C. Fort, P. Maddaloni, M. Inguscio, A. Smerzi, A. Trombettoni, P.G. Kevrekidis, and A.R. Bishop. A novel mechanism for superfluidity breakdown in weakly coupled Bose-Einstein condensates. arXiv:cond-mat/0207139, 2002. 
21. P. L. Christiansen, Yu. B. Gaididei, M. Johansson, and K. Ø. Rasmussen. Breatherlike excitations in discrete lattices with noise and nonlinear damping. Phys. Rev. B, 55:5759-5766, 1997.

22. P. L. Christiansen, Yu. B. Gaididei, K. Ø. Rasmussen, V. K. Mezentsev, and J. Juul Rasmussen. Dynamics in discrete two-dimensional nonlinear Schrödinger equations in the presence of point defects. Phys. Rev. B, 54:900912, 1996.

23. D N Christodoulides and R I Joseph. Discrete self-focussing in nonlinear arrays of coupled waveguides. Optics Letters, 13:794-796, 1988.

24. Ch. Claude, Yu.S. Kivshar, O. Kluth, and K.H. Spatschek. Moving localized modes in nonlinear lattices. Phys. Rev. B, 47:14228-14232, 1993.

25. L Cruzeiro-Hansson, H Feddersen, R Flesch, P L Christiansen, M Salerno, and A C Scott. Classical and quantum analysis of chaos in the discrete selftrapping equation. Phys. Rev. B, 42(1):522-526, 1990.

26. S. Darmanyan, A. Kobyakov, and F.Lederer. Asymmetric dark solitons in nonlinear lattices. JETP, 93:429-434, 2001.

27. S. Darmanyan, A. Kobyakov, and F. Lederer. Stability of strongly localized excitations in discrete media with cubic nonlinearity. JETP, 86:682-686, 1998.

28. I. Daumont, T. Dauxois, and M. Peyrard. Modulational instability: first step towards energy localization in nonlinear lattices. Phys. Rev. A, 46:3198-3205, 1996.

29. A.S. Davydov. The theory of contraction of proteins under their excitation. J. Theor. Biol., 38:559-569, 1973.

30. D. B. Duncan, J. C. Eilbeck, H. Feddersen, and J. A. D. Wattis. Solitons on lattices. Physica D, 68:1-11, 1993.

31. J. C. Eilbeck. Numerical simulations of the dynamics of polypeptide chains and proteins. In Chikao Kawabata and A. R. Bishop, editors, Computer Analysis for Life Science - Progress and Challenges in Biological and Synthetic Polymer Research, pages 12-21, Tokyo, 1986. Ohmsha.

32. J. C. Eilbeck. Nonlinear vibrational modes in a hexagonal molecule. In A. Davydov, editor, Physics of Many-Particle Systems, volume 12, pages 41-51, Kiev, 1987.

33. J. C. Eilbeck, P. S. Lomdahl, and A. C. Scott. Soliton structure in crystalline acetanilide. Phys. Rev. B, 30:4703-4712, 1984.

34. J. C. Eilbeck, P. S. Lomdahl, and A. C. Scott. The discrete self-trapping equation. Physica D, 16:318-338, 1985 .

35. J. C. Eilbeck and A C Scott. Theory and applications of the discrete selftrapping equation. In P. L. Christiansen and R D Parmentier, editors, Structure, Coherence, and Chaos in Dynamical Systems, pages 139-159. Manchester University Press, 1989.

36. H.S. Eisenberg, Y. Silberberg, R. Morandotti, A.R. Boyd, and J.S. Aitchison. Discrete spatial optical solitons in waveguide arrays. Phys. Rev. Lett., 81:3383-3386, 1998.

37. L. D. Faddeev and L. A. Takhtajan. Hamiltonian methods in the theory of solitons. Springer-Verlag, Berlin, 1987.

38. H. Feddersen. Localization of vibrational-energy in globular protein. Physics 
Letters A, 154:391-395, 1991.

39. H. Feddersen. Solitary wave solutions to the discrete nonlinear Schrödinger equation. In M. Remoissenet and M. Peyrard, editors, Nonlinear Coherent structures in Physics and Biology, v. 393 of Lecture Notes in Physics, pages 159-167. Springer, 1991.

40. H. Feddersen. Numerical calculations of solitary waves in Davydov's equations. Physica Scripta, 47:481-483, 1993.

41. S. Flach and K. Kladko. Moving discrete breathers? Physica D, 127:61-72, 1999.

42. S. Flach, K. Kladko, and R.S. MacKay. Energy thresholds for discrete breathers in one-, two-, and three-dimensional lattices. Phys. Rev. Lett., 78:1207-1210, 1997.

43. S. Flach and C. R. Willis. Discrete breathers. Phys. Rep., 295:182-264, 1998.

44. S. Flach, Y. Zolotaryuk, and K. Kladko. Moving lattice kinks and pulses: An inverse method. Phys. Rev. E, 59:6105-6115, 1999.

45. J. Fröhlich, T. Spencer, and C.E. Wayne. Localization in disordered, nonlinear dynamical systems. J. Stat. Phys., 42:247-274, 1986.

46. Y. B. Gaididei, S. F. Mingaleev, and P. L. Christiansen. Curvature-induced symmetry breaking in nonlinear Schrödinger models. Phys Rev E, 62:R53R56, 2000.

47. D. Hennig and H. Gabriel. The 4-element discrete nonlinear Schrödinger equation - non-integrability and Arnold diffusion. J. Phys. A, 28:3749-3756, 1995.

48. D. Hennig, H. Gabriel, M. F. Jørgensen, P. L. Christiansen, and C. B. Clausen. Homoclinic chaos in the discrete self-trapping trimer. Phys. Rev. E, 51:2870-2876, 1995.

49. D. Hennig, K. O. Rasmussen, G. P. Tsironis, and H. Gabriel. Breatherlike impurity modes in discrete nonlinear lattices. Physical Review E, 52:R4628R4631, 1995.

50. D. Hennig and G. P. Tsironis. Wave transmission in nonlinear lattices. Phys. Rep., 307:334-432, 1999.

51. T. Holstein. Studies of polaron motion. Ann. Phys., 8:325-389, 1959.

52. A. G. Izergin and V. E. Korepin. A lattice model related to the nonlinear Schrödinger equation. Sov. Phys. Dokl., 26:653-654, 1981.

53. S. M. Jensen. The nonlinear coherent coupler. IEEE J. Quantum Electron., QE-18:1580-1583, 1982.

54. M. Johansson. Decay of discrete nonlinear Schrödinger breathers through inelastic multiphonon scattering. Phys. Rev. E, 63:037601-1-4, 2001.

55. M. Johansson and S. Aubry. Existence and stability of quasiperiodic breathers in the discrete nonlinear Schrödinger equation. Nonlinearity, 10:1151-1178, 1997.

56. M. Johansson and S. Aubry. Growth and decay of discrete nonlinear Schrödinger breathers interacting with internal modes or standing-wave phonons. Phys Rev E, 61:5864-5879, 2000.

57. M. Johansson, S. Aubry, Yu. B. Gaididei, P. L. Christiansen, and K. Ø. Rasmussen. Dynamics of breathers in discrete nonlinear Schrödinger models. Physica D, 119:115-124, 1998. 
58. M. Johansson, M. Hörnquist, and R. Riklund. Effects of nonlinearity on the time evolution of single-site localized states in periodic and aperiodic discrete systems. Phys. Rev. B, 52:231-240, 1995.

59. M. Johansson and Yu.S. Kivshar. Discreteness-induced oscillatory instabilities of dark solitons. Phys. Rev. Lett., 82:85-88, 1999.

60. M. Johansson, A.M. Morgante, S. Aubry, and G. Kopidakis. Standing wave instabilities, breather formation and thermalization in a Hamiltonian anharmonic lattice. Eur. Phys. J. B, 29:279-283, 2002.

61. G. Kalosakas, K. Ø. Rasmussen, and A. R. Bishop. Delocalizing transition of Bose-Einstein condensates in optical lattices. Phys Rev Lett, 89:030402-1-4, 2002.

62. P. G. Kevrekidis, B. A. Malomed, and Yu. B. Gaididei. Solitons in triangular and honeycomb dynamical lattices with the cubic nonlinearity. Phys Rev E, 66:016609-1-10, 2002.

63. P. G. Kevrekidis, K. Ø. Rasmussen, and A. R. Bishop. The discrete nonlinear Schrödinger equation: a survey of recent results. Int J Mod Phys B, 15:28332900, 2001.

64. S.W. Kim and S. Kim. The structure of eigenmodes and phonon scattering by discrete breathers in the discrete nonlinear Schrödinger chain. Physica D, 141:91-103, 2000.

65. Y. S. Kivshar and D. K. Campbell. Peierls-Nabarro potential barrier for highly localized nonlinear modes. Physical Review E, 48:3077-3081, 1993.

66. Y. S. Kivshar, W. Krolikowski, and O. A. Chubykalo. Dark solitons in discrete lattices. Physical Review E, 50:5020-5032, 1994.

67. Yu.S. Kivshar, D.E. Pelinovsky, T. Cretegny, and M. Peyrard. Internal modes of solitary waves. Phys. Rev. Lett., 80:5032-5035, 1998.

68. Yu.S. Kivshar and M. Peyrard. Modulational instabilities in discrete lattices. Phys. Rev. A, 46:3198-3205, 1992.

69. G. Kopidakis and S. Aubry. Intraband discrete breathers in disordered nonlinear systems. I. Delocalization. Physica D, 130:155-186, 1999.

70. G. Kopidakis and S. Aubry. Discrete breathers and delocalization in nonlinear disordered systems. Phys. Rev. Lett., 84:3236-3239, 2000.

71. G. Kopidakis and S. Aubry. Intraband discrete breathers in disordered nonlinear systems. II. Localization. Physica D, 139:247-275, 2000.

72. E. W. Laedke, K. H. Spatschek, V. K. Mezentsev, S. L. Musher, I. V. Ryzhenkova, and S. K. Turitsyn. Instability of 2-dimensional solitons in discrete-systems. JETP Letters, 62:677-684, 1995.

73. E. W. Laedke, K. H. Spatschek, and S. K. Turitsyn. Stability of discrete solitons and quasicollapse to intrinsically localized modes. Physical Review Letters, 73:1055-1059, 1994.

74. S.-S. Lee and S. Kim. Phonon scattering by breathers in the discrete nonlinear Schrödinger chain. Int. J. Mod. Phys. B, 14:1903-1914, 2000.

75. R. S. MacKay and S. Aubry. Proof of existence of breathers for time-reversible or Hamiltonian networks of weakly coupled oscillators. Nonlinearity, 7:16231643, 1994.

76. B.A. Malomed and P.G. Kevrekidis. Discrete vortex solitons. Phys. Rev. E, 64:026601-1-6, 2001. 
77. V. K. Mezentsev, S. L. Musher, I. V. Ryzhenkova, and S. K. Turitsyn. 2dimensional solitons in discrete-systems. Jetp Letters, 60:829-835, 1994.

78. M. I. Molina and G. P. Tsironis. Dynamics of self-trapping in the discrete nonlinear Schrödinger-equation. Physica D, 65:267-273, 1993.

79. M. I. Molina and G. P. Tsironis. Absence of localization in a nonlinear random binary alloy. Physical Review Letters, 73:464-467, 1994.

80. R. Morandotti, H.S. Eisenberg, Y. Silberberg, M. Sorel, and J.S. Aitchison. Self-focusing and defocusing in waveguide arrays. Phys. Rev. Lett., 86:32963299, 2001.

81. R. Morandotti, U. Peschel, J.S. Aitchison, H.S. Eisenberg, and Y. Silberberg. Dynamics of discrete solitons in optical waveguide arrays. Phys. Rev. Lett., 83:2726-2729, 1999.

82. R. Morandotti, U. Peschel, J.S. Aitchison, H.S. Eisenberg, and Y. Silberberg. Experimental observation of linear and nonlinear optical Bloch oscillations. Phys. Rev. Lett., 83:4756-4759, 1999.

83. A. M. Morgante, M. Johansson, G. Kopidakis, and S. Aubry. Standing wave instabilities in a chain of nonlinear coupled oscillators. Physica D, 162:53-94, 2002.

84. T. Pertsch, P. Damberg, W. Elflein, A. Bräuer, and F. Lederer. Optical Bloch oscillations in temperature tuned waveguide arrays. Phys. Rev. Lett., 83:4752-4755, 1999.

85. T. Pertsch, U. Peschel, and F. Lederer. Hybrid discrete solitons. Phys. Rev. E., 66:Scheduled 1 December, 2002.

86. M. Peyrard. The pathway to energy localization in nonlinear lattices. Physica D, 119:184-199, 1998.

87. J. Juul Rasmussen and K. Rypdal. Blow-up in Nonlinear Schroedinger equations. Physica Scripta., 33:481-504, 1986.

88. K.Ø. Rasmussen, S. Aubry, A.R. Bishop, and G.P. Tsironis. Discrete nonlinear Schrödinger breathers in a phonon bath. Eur. Phys. J. B, 15:169-175, 2000 .

89. K.Ø. Rasmussen, T. Cretegny, P.G. Kevrekidis, and N. Grønbech-Jensen. Statistical mechanics of a discrete nonlinear system. Phys. Rev. Lett., 84:3740-3743, 2000.

90. M Salerno. Quantum deformations of the discrete nonlinear Schrödinger equation. Phys. Rev. A, 46:6856-6859, 1992.

91. A. C. Scott. Davydov's soliton. Phys. Rep., 217:1-67, 1992.

92. A. C. Scott. Nonlinear Science. OUP, Oxford, 1999.

93. A C Scott and J C Eilbeck. On the CH stretch overtones in benzene. Chem. Phys. Lett., 132:23-28, 1986.

94. A. C. Scott and L. MacNeil. Binding energy versus nonlinearity for a "small" stationary soliton. Phys. Lett. A, 98:87-88, 1983.

95. D.L. Shepelyansky. Delocalization of quantum chaos by weak nonlinearity. Phys. Rev. Lett., 70:1787-1790, 1993.

96. A. Smerzi, S. Fantoni, S. Giovanazzi, and S.R. Shenoy. Quantum coherent atomic tunneling between two trapped Bose-Einstein condensates. Phys. Rev. Lett., 79:4950-4953, 1997.

97. A. Trombettoni and A. Smerzi. Discrete solitons and breathers with dilute 
Bose-Einstein condensates. Phys. Rev. Lett., 86:2353-2356, 2001.

98. A.A. Vakhnenko and Yu.B. Gaididei. On the motion of solitons in discrete molecular chains. Theor. Math. Phys., 68:873-880, 1987.

99. M. I. Weinstein. Excitation thresholds for nonlinear localized modes on lattices. Nonlinearity, 12:673-691, 1999. 